\title{
Copper efflux transporter (ATP7B) contributes to the acquisition of cisplatin-resistance in human oral squamous cell lines
}

\author{
KUNIO YOSHIZAWA, SHINICHI NOZAKI, HIROKO KITAHARA, TERUHISA OHARA, \\ KOROKU KATO, SHUICHI KAWASHIRI and ETSUHIDE YAMAMOTO
}

\author{
Department of Oral and Maxillofacial Surgery, Kanazawa University Graduate School of Medical Science, \\ 13-1 Takaramachi, Kanazawa, Ishikawa 920-8641, Japan
}

Received February 2, 2007; Accepted June 14, 2007

\begin{abstract}
Acquired resistance to cisplatin (CDDP) is an issue in cancer chemotherapy. This resistance has been reported to be correlated with the expression of the $\mathrm{Cu}$ influx copper transporter 1 (CTR1) and two copper efflux transporters (ATP7A, ATP7B). We investigated the correlation between the expression of these transporters and the sensitivity to CDDP using three pairs of parent cell lines and resistant cell lines derived from various types of invasive oral squamous cell carcinoma (OSCC). Using multiple steps, each of the CDDPresistant cell lines, HSC-4-R, OSC-19-R, HOC313-R, was selected from HSC-4 cells derived from a cancer with medium invasiveness, OSC-19 cells derived from a cancer with high invasiveness and HOC 313 cells derived from a cancer with the highest invasiveness. Resistant cell lines had a stronger expression of ATP7B in conjunction with the acquisition of CDDP-resistance than parent cell lines. Furthermore, OSC19-R cells transfected with the ATP7B siRNA had a 10.6-fold higher sensitivity to CDDP compared to OSC-19-R cells transfected with a nonsense siRNA. These results suggest that each of the resistant cell lines had acquired resistance to CDDP due to the overexpression of ATP7B. On the other hand, the expression of CTR1 was the same between sensitive cell lines and resistant cell lines and ATP7A mRNA expression was barely noted. We conclude that ATP7B is correlated with the
\end{abstract}

Correspondence to: Dr Kunio Yoshizawa, Department of Oral and Maxillofacial Surgery, Kanazawa University Graduate School of Medical Science, 13-1 Takaramachi, Kanazawa, Ishikawa 920-8641, Japan

E-mail: yoshizawa@oral.m.kanazawa-u.ac.jp

Abbreviations: OSCC, oral squamous cell carcinoma; CDDP, cisplatin; PPC, peak plasma concentration; RT-PCR, reverse transcription-polymerase chain reaction; MTS, 3-(4,5dimethylthiazol-2-yl)-5-(3-carboxymethoxyphenyl)-2-(4sulfophenyl)-2H-tetrazolium inner salt; MEM, minimal essential medium; MDR, multidrug resistance; ABC, ATP-binding cassette; EMT, epithelial-to-mesenchymal transition

Key words: multidrug resistance, oral cancer, epithelial-tomesenchymal transition acquisition of CDDP resistance more closely than either CTR1 or ATP7A. ATP7B may be a key determinant in the acquired resistance to CDDP in OSCC.

\section{Introduction}

Oral squamous cell carcinoma (OSCC) is the most common histologic subtype of oral malignancy. Cisplatin (CDDP) and other platinum compounds are the most clinically effective chemotherapeutic agents used for the treatment of OSCC. Acquired resistance to CDDP is an issue in cancer chemotherapy $(1,2)$. Although the administration of CDDP before and after surgery improves the prognosis of patients with OSCC, the 5-year survival rates are still not satisfactory $(1,3)$.

Further knowledge on the mechanism of drug sensitivity and resistance may lead to new strategies that would avoid resistance to chemotherapy and the side-effects of chemotherapy. The main mechanisms that lead to clinical resistance to CDDP have not been well defined $(4,5)$. Currently, acquired resistance to CDDP is believed to be multifactorial and involves reduced accumulation, increased cytoplasmic detoxification and an increase in repair of damaged DNA. However, impaired accumulation of the drug is the most commonly observed difference between resistant cells and the drug-sensitive cells from which they were derived (5-7). It has been reported that the transporter by which CDDP enters or exits from cells includes human copper transporter 1 (CTR1) for the intake and two copper export transporters, ATP7A and ATP7B (8-14). To date, strong evidence has been collected that these transporters regulate the sensitivity to platinumcontaining drugs and control their accumulation levels; however, there is no general agreement as to which transporter is the most responsible (8)

A direct link between copper transporters and CDDP resistance has been demonstrated by Komatsu et al (12), who found that prostrate cancer cells resistant to CDDP expressed an increased level of ATP7B, which has been reported to effect the efflux of CDDP in the trans-Golgi network and that the cells transfected with ATP7B are resistant to both copper and CDDP. Increased levels of ATP7B mRNA or protein have been found in several major human malignancies, including ovarian cancer, gastric carcinoma, breast cancer and OSCC (2,8,11-14). Research has also suggested that a high ATP7B expression level in OSCC was associated with an unfavorable 
clinical outcome in patients treated with CDDP-based chemotherapy (1).

To understand the clinically relevant mechanisms of cellular sensitivity to CDDP and to help develop new treatments for carcinomas with acquired CDDP resistance, we examined the biochemical and molecular alterations of transporter expression in the parent and resistant cell lines derived from various invasive OSCCs.

\section{Materials and methods}

Drugs. CDDP was generously provided by Nippon Kayaku (Tokyo, Japan) and stored as a $0.5 \mathrm{mg} / \mathrm{ml}$ stock solution in $0.9 \% \mathrm{NaCl}$ at room temperature with light shielding.

Cell culture and cell lines. OSCC parent cell lines, HSC-4, OSC-19 and HOC313 and their CDDP-resistant cell lines, HSC-4-R, OSC-19-R and HOC313-R on 100-mm plate, were maintained at $37^{\circ} \mathrm{C}$ in a humidified incubator containing $5 \%$ $\mathrm{CO}_{2}$ in a minimal essential medium (MEM) (Sigma-Aldrich, Ayrshire, UK) supplemented with $10 \%$ fetal bovine serum and $1 \%$ penicillin-streptomycin. The cell lines were derived from OSCCs with the following grades of invasiveness, HSC-4 from a mildly invasive type; OSC-19 from a highly invasive type; and HOC313 from the most highly invasive type (15).

Establishment of CDDP-resistant cell lines. CDDP was then added to the medium with increasing concentrations. CDDPresistant variants of the cell lines were isolated using stepwise selection by increasing the CDDP concentrations, starting with the dose required to reduce the surviving fraction to $10 \%$ of the initial fraction for each cell line $\left(\mathrm{IC}_{90}\right)$. When the cells became confluent in the medium containing CDDP, the drug concentration was increased to $2 \times \mathrm{IC}_{90}, 3 \times \mathrm{IC}_{90}, 5 \times \mathrm{IC}_{90}$ and finally $10 \times \mathrm{IC}_{90}$, which was the maximal concentration used. The cells were continuously exposed for 4 to 8 weeks to each drug concentration. During continuous exposure to CDDP, the culture medium was replaced with a freshly prepared medium containing CDDP at indicated concentrations every 3 days. Subculture was continued once a week and took 30 weeks to make all the resistant cell lines. In the present experiment, we did not use any mutagens.

Determination of drug sensitivity. The sensitivity of the cells to the drugs was assessed using an 3-(4,5-dimethylthiazol-2-yl)5-(3-carboxy methoxyphenyl)-2-(4-sulfophenyl)-2Htetrazolium, inner salt (MTS) colorimetric assay as follows. Exponentially growing cells $\left(3 \times 10^{3} /\right.$ well in $100 \mu 1$ of MEM) were seeded in 96-well plates. The cells were immediately mixed with $100 \mu \mathrm{l}$ of MEM containing 0.42-83 $\mu \mathrm{M}$ CDDP, which was a concentration equivalent to $0.05-10 \mathrm{x}$ peak plasma concentration (PPC) based on acute animal toxicology data; the cells were then incubated for 3 days. After 3 days of culture, $20 \mu 1$ of CellTiter 96 AQueous One Solution (Promega, Madison, WI, USA) was added and after $1 \mathrm{~h}$, the absorbance in each well was measured at $490 \mathrm{~nm}$ using a model 680 spectrophotometer (Bio-Rad, Hercules, CA, USA). The $\mathrm{IC}_{50}$ was measured as the concentration of the compound that reduced the number of cells to $50 \%$ of that in the control medium.
Table I. Sensitivity of OSCC lines after $72 \mathrm{~h}$ of CDDP exposure.

\begin{tabular}{lccc}
\hline Cell type & $\begin{array}{c}\mathrm{IC}_{50}, \mu \mathrm{M} \\
(\text { mean } \pm \mathrm{SD})\end{array}$ & $\begin{array}{c}\text { Fold } \\
\text { resistance }\end{array}$ & $P$ \\
\hline HSC-4 & $2.6 \pm 0.4$ & - & - \\
HSC-4-R & $6.0 \pm 1.6$ & 2.4 & $<0.05$ \\
OSC-19 & $6.1 \pm 1.0$ & - & - \\
OSC-19-R & $30.6 \pm 6.3$ & 5.0 & $<0.01$ \\
HOC313 & $6.0 \pm 0.4$ & - & - \\
HOC313-R & $43.8 \pm 9.4$ & 7.3 & $<0.01$ \\
\hline
\end{tabular}

Fold resistance was determined by dividing the $\mathrm{IC}_{50}$ value of the CDDP for the resistant cells by that of the parent cells. There was a significant association between all of the pairs of parent and resistant cell lines. The $\mathrm{IC}_{50}$ values are presented as the mean of triplicate \pm S.D.

RNA extraction and reverse transcriptase-polymerase chain reaction ( $R T-P C R)$. RNA was extracted from $80 \%$ confluent cultured parent and resistant cell lines using an RNeasy Kit (Qiagen). A $1 \mu \mathrm{g}$ sample in $10 \mu \mathrm{l}$ of RNase free water was incubated for $5 \mathrm{~min}$ at $60^{\circ} \mathrm{C}$ and then quickly chilled on ice for $5 \mathrm{~min}$. The RNA samples were reverse-transcribed into firststrand cDNA at $40^{\circ} \mathrm{C}$ for $40 \mathrm{~min}$ in RT solution. The cDNA samples were amplified by the following primers $(5,14,16)$ : for the CTR1, 5'-agc tat atg gac tcc aac ag-3' (forward) and 5'-cgt tgt agg tca tga aga tg-3' (reverse); for ATP7A, 5'-gcc tgc gta cgt gga ttt at-3' (forward) and 5'-tca atg gtc caa aca cag ga-3' (reverse); for ATP7B, 5'-tcc tgg tgg cta ttg acg gtg t-3' (forward) and 5'-cat tca ggc gca gag acc act t-3' (reverse); and for $\beta$-actin, 5'-gaa aat ctg gca cca cac ctt-3' (forward) and 5'-ttg aag gta gtt tcg tgg at-3' (reverse). PCRs were carried out under the following conditions: $3 \mathrm{~min}$ at $94^{\circ} \mathrm{C}$, followed by cycles (26 for CTR1, 40 for ATP7A, 34 for ATP7B and 20 for Bactin) of $1 \mathrm{~min}$ at $94^{\circ} \mathrm{C}, 1 \mathrm{~min}$ at $54^{\circ} \mathrm{C}$ and $1 \mathrm{~min}$ at $72^{\circ} \mathrm{C}$. All reactions were completed with a final incubation at $72^{\circ} \mathrm{C}$ for $10 \mathrm{~min}$. The lengths of the amplified fragments for CTR 1 , ATP7A, ATP7B and B-actin genes were 445, 156, 883 and 592 bp, respectively. PCR products were detected using $1.5 \%$ agarose gel electrophoresis and staining with ethidium bromide. The mRNA levels of CTR1, ATP7A and ATP7B were analyzed by RT-PCR and were normalized by the B-actin signal.

Western blot analysis. Parent and resistant cells on each of $80 \%$ confluent plates were used as protein samples. The protein samples $(50 \mu \mathrm{g})$ that were extracted from the whole cellular structure using M-PER Mammalian protein extraction reagent (Pierce, Rockford, IL, USA) were heated at $95^{\circ} \mathrm{C}$ for $5 \mathrm{~min}$ before electrophoresis and then subjected to $10 \%$ SDSPAGE. After electrophoresis, the samples were transferred onto PVDF membranes (ATTO Co, Tokyo, Japan) and incubated for $1 \mathrm{~h}$ with 200-fold diluted polyclonal anti-rabbit antibody against ATP7B (Novus, Littleton, CO, USA) and 5000 -fold diluted polyclonal anti-mouse antibody $\beta$-actin (Sigma, St. Louis, MO, USA) respectively. The membrane was washed three times with PBS and then incubated for $1 \mathrm{~h}$ with 2000-fold diluted horseradish peroxidase-conjugated anti- 


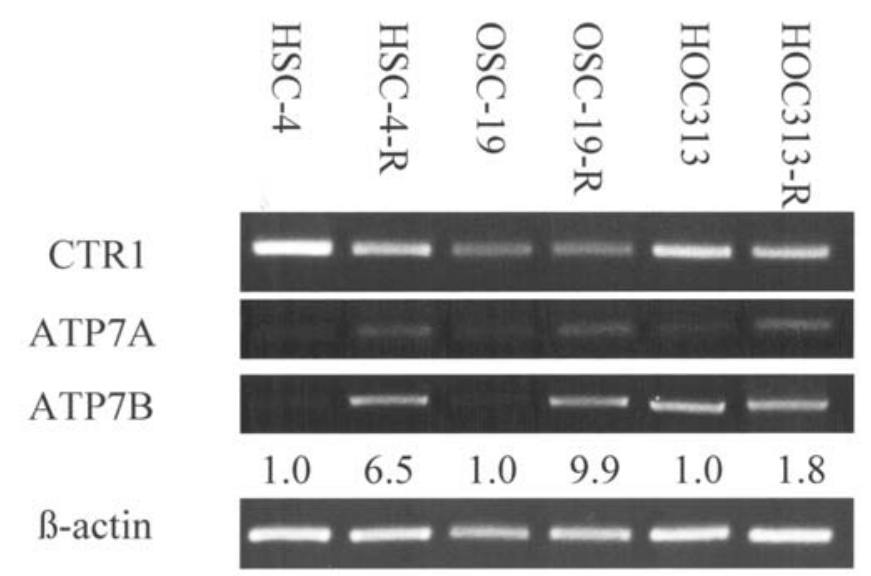

Figure 1. Comparison of transporter mRNA levels (CTR1, ATP7A and ATP7B) between each of the CDDP parent and resistant cells using RT-PCR. These cell lines showed low ATP7A mRNA expression levels and equivalent levels of CTR1. In contrast, ATP7B expression was higher in resistant cells than in the parent cell lines.

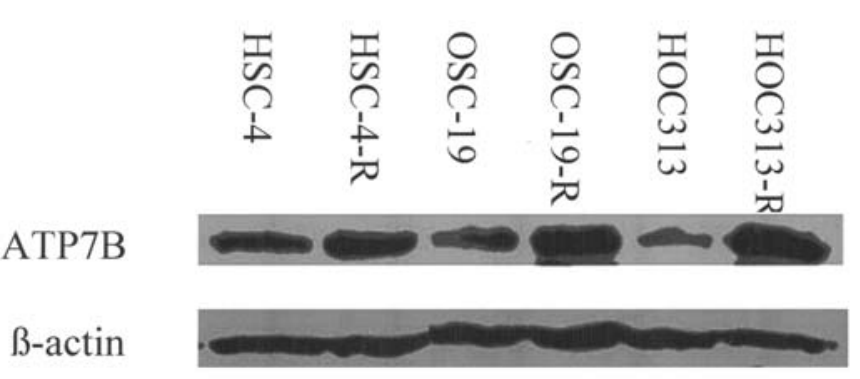

Figure 2. ATP7B protein levels in CDDP parent and resistant cells on Western blot analysis. ATP7B protein expression was stronger in the resistant cell lines than in the parent cell lines.

rabbit IgG (Amersham, Little Chalfont, Buckinghamshire, England) to detect ATP7B and 2000-fold diluted horseradish peroxidase-conjugated anti-mouse IgG (Amersham) to detect B-actin, respectively. The blots were revealed by enhanced chemiluminescent detection carried out according to the manufacturer's instructions.

siRNA transfection. siRNA transfection was done as described previously by Nozaki et al (16). Two oligoribonucleotides (5'-AAUUGAUAUUGAGCGGUUA-3' and 5'-UAACCGGU CAAUAUCAAUU-3') were used to inhibit ATP7B synthesis (Qiagen). As a control, we used two nonsense siRNA oligoribonucleotides: 5'-UUCUCCGAACGUGUCACGU-3' and 5'-AUGUGACACGUUCGGAGAA-3'. The cells were transfected with the oligoribonucleotides using Hyperfect Transfection Reagent (Qiagen). The transfection was done for $48 \mathrm{~h}$ on subconfluent cells, with a final siRNA concentration of $5 \mathrm{nM}$. In each experiment, a series of cells were used for RT-PCR to evaluate ATP7B inhibition.

Statistics. Results are representative of at least three experiments. The statistical significance of the differences between groups was evaluated using a single two-tailed Student's t-test.
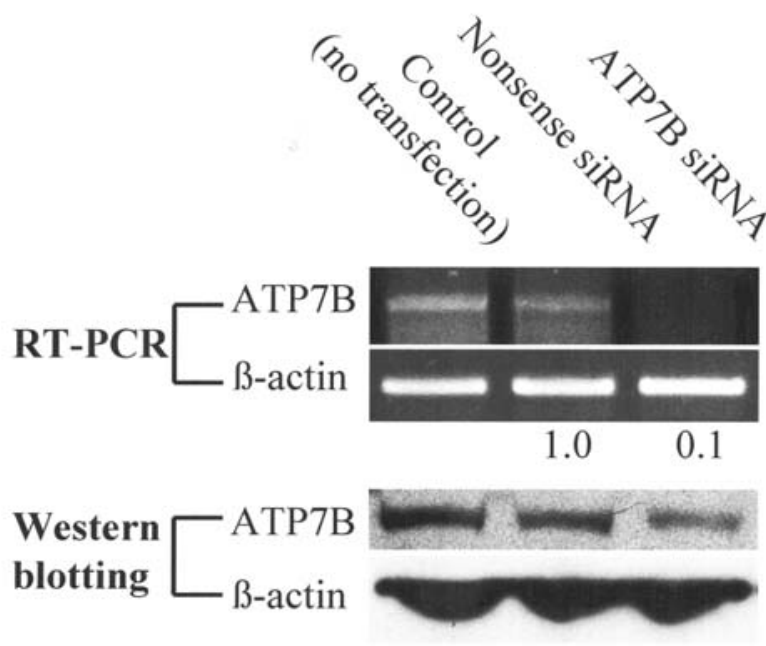

Figure 3. Suppression of ATP7B mRNA and protein of OSC-19-R with an ATP7B siRNA. RT-PCR and Western blotting showed that expression of ATP7B mRNA and protein was decreased after siRNA transfection.

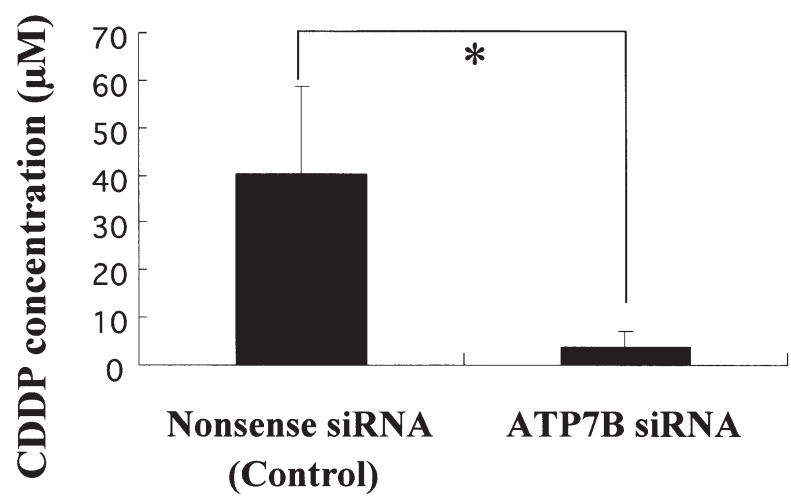

Figure 4. Effect of silencing ATP7B on CDDP sensitivity of OSC-19-R. Cell viability was assessed by the MTS assay as described under Materials and methods. The $\mathrm{IC}_{50}(3.8 \mu \mathrm{M})$ of the OSC-19-R cells transfected with the ATP7B siRNA had significantly 10.6-fold higher sensitivity to CDDP compared to the $\mathrm{IC}_{50}(40.1 \mu \mathrm{M})$ of the OSC-19-R cells transfected with a nonsense siRNA. The bars represent the means of determination for four times and the error bars represent the SD. ${ }^{*} \mathrm{p}<0.01$.

\section{Results}

Sensitivity of OSCC cell lines to CDDP. The $\mathrm{IC}_{50}$ of the $\mathrm{CDDP}$ for each cell line was determined using a colorimetric MTS assay (Table I). Fold resistance was determined by dividing the $\mathrm{IC}_{50}$ value of the CDDP for resistant cells by that of the parent cells. CDDP-resistant cell lines HSC-4-R, OSC-19-R, HOC313-R were 2.4-, 5.0-, and 7.3-fold more resistant than the parental cells, respectively. There was a significant association at each $\mathrm{IC}_{50}$ value between all pairs of the parent and resistant cell lines.

The levels of CTR1, ATP7A and ATP7B $m R N A$ in the three pairs of parent- and resistant- cell lines by RT-PCR. CTR1 was slightly decreased in resistant cells such as HSC-4-R and HOC313-R (Fig. 1). In addition, though these cell lines expressed little ATP7A mRNA, the expression of ATP7A and 
ATP7B was apparently elevated in resistant cells. Based on these results ATP7B most strongly changed between parent and resistant cell lines. The expression of ATP7B of CDDP resistant cell lines HSC-4-R, OSC-19-R, HO313-R were 6.5-, 9.9- and 1.8-fold more than that of parent cell lines (Fig. 1). Compared to the parent cell line, the expression of ATP7B in HOC 313 cells was higher than in HSC-4 and OSC-19 cells.

Expression of ATP7B protein. The level of ATP7B protein expression was examined using the Western blotting method (Fig. 2). The expression of ATP7B protein was stronger in resistant cell lines than in parent cell lines. Furthermore, cell lines, such as OSC-19 and HOC313, which had a high acquired resistance to CDDP, exhibited greater expression of ATP7B protein in the process of acquiring CDDP-resistance than cell lines, such as HSC-4, that had low acquired resistance to CDDP. The protein level of ATP7B in HSC-4 is the highest, whereas it is the most CDDP-sensitive cell line (Fig. 2).

CDDP sensitivity of OSC-19-R cells with siRNA-silenced $A T P 7 B$. Next, we examined the effect of reducing the expression of ATP7B mRNA in OSC-19-R cells using siRNA. The reason why we used OSC-19-R in this procedure is that it exhibited the strongest expression of ATP7B protein and mRNA level in the process of acquiring CDDP-resistance than other cell lines. RT-PCR showed that the level of ATP7B mRNA was 90\% lower in OSC-19-R cells transfected with the ATP7B siRNAs than in OSC-19-R cells transfected with a nonsense siRNA (Fig. 3). The sensitivity to CDDP of the transfected cells was assessed using the MTS colorimetric assay as identified above. The $\mathrm{IC}_{50}(3.8 \mu \mathrm{M})$ of the OSC-19-R cells transfected with the ATP7B siRNA had significantly 10.6-fold higher sensitivity to CDDP compared to the $\mathrm{IC}_{50}(40.1 \mu \mathrm{M})$ of the OSC-19-R cells transfected with a nonsense siRNA (Fig. 4).

\section{Discussion}

In the current experiments, we showed that compared to parent cell lines, resistant cell lines exhibited a 2.4 to 7.3 -fold resistance to CDDP and had a stronger expression of ATP7B than the expression of other transporters when they acquired CDDP resistance. Furthermore, the $\mathrm{IC}_{50}$ of OSC-19-R cells transfected with the ATP7B siRNA had 10.6-fold higher sensitivity to CDDP compared to OSC-19-R cells transfected with a nonsense siRNA. This is the first study to report that repression of ATP7B with siRNA raises the sensitization effects to CDDP in acquired CDDP-resistant cell lines. These results suggest that each of the resistant cell lines acquired resistance to CDDP due to overexpression of ATP7B, which resulted in a greater efflux of CDDP. However, these in vitro results cannot be directly applied in vivo, since, in clinical practice, changes in the sensitivity of cells to chemotherapeutic agents are mediated by a variety of biotransformations. The protein level of ATP7B in HSC-4 is the highest, whereas it is the most CDDP-sensitive cell line (Fig. 2). This discrepancy could be explained by not only the expression of strength and weakness of ATP7B protein on Western blotting, but that the modification including methylation may go on activating ATP7B as efflux transporter of CDDP in vivo.
The discovery of the multidrug resistance (MDR) transporter that was produced from the ATP-binding cassette $(\mathrm{ABC})$ gene opened a new avenue for understanding the origins of drug resistance (17). It is now well established that multidrug resistant proteins (MRP) play an important role in the tumor cell drug resistance in vitro and in vivo (17). It was recently reported that MRP2 (multidrug resistance protein 2), also known as the canalicular multispecific organ anion transporter (cMOAT), is overexpressed in a number of CDDPresistant cell lines (18-20). However, in vitro data suggest that MRP2 acts as a drug efflux pump and there is no evidence that it is involved in the drug resistance clinically seen in oral cancer patients. We did not find that MRP2 mRNA was expressed in OSCC cell lines using RT-PCR (data not shown). Our results suggest that the copper transporter ATP7B, which is a member of the heavy metal-transporting, P-type ATPases and which includes the ATP-binding domain of the transmembrane segment, plays a more important role than other transporters in the influx and efflux of CDDP.

Activation of NF- $\mathrm{NB}$ has been shown to induce resistance through the expression of the MDR1 gene (21). Furthermore, the inhibition of $\mathrm{NF}-\kappa \mathrm{B}$ has been shown to increase the potency of CDDP both in vitro and in vivo (22). Transcriptional factors, including NF-кB, may control the CDDP pathway by promoting not only MDR1 but also the expression of ATP7B, which belongs to the same ABC transporter family as MDR1. Furthermore, there are studies dealing with in vitro/in vivo models of mammary carcinoma that have reported that NF- $\mathrm{\kappa B}$ was essential for both the induction and the maintenance of the epithelial-to-mesenchymal transition (EMT) and invasion (23-25). In this connection, it was reported that EMT reflects an important process by which colorectal cancer cells may potentially acquire chemoresistance to platinum compounds (26). The HOC313 cell lines showed characteristics of EMT, including spindle-cell shape, loss of polarity, intercellular separation, decreased expression of the epithelial adhesion molecule E-cadherin and increased expression of vimentin as a mesenchymal marker on RT-PCR; other invasive grade cell lines did not show these characteristics (data not shown) (27). Therefore, HOC 313 cells that acquired a high resistance to CDDP appeared to have the characteristics of EMT that were similar to colorectal cancer cells.

In conclusion, methods targeting the transcriptional element NF- $\mathrm{KB}$ and ATP7B could be useful complementary therapies for reducing the invasiveness of OSCCs and CDDP resistance.

\section{Acknowledgements}

The authors would like to thank all members of the department for their helpful suggestions and support. This study was supported by science research grant (no. 17390533) provided by the Ministry of Education, Science, Sports and Culture of Japan.

\section{References}

1. Miyashita H, Nitta Y, Mori S, et al: Expression of coppertransporting P-type adenosine triphosphatase (ATP7B) as a chemoresistance marker in human oral squamous cell carcinoma treated with cisplatin. Oral Oncol 39: 157-162, 2003. 
2. Fujii R, Mutoh M, Niwa K, et al: Active efflux system for cisplatin-resistant human KB cells. Jpn J Cancer Res 85: 426-433, 1994.

3. Hill BT and Price LA: Lack of survival advantage in patients with advanced squamous cell carcinomas of the oral cavity receiving neoadjuvand chemotherapy prior to local therapy, despite of achieving an initial high clinical complete remission rate. Am J Clin Oncol 17: 1-5, 1994.

4. Samimi G, Safaei R, Katano K, et al: Increased expression of the copper efflux transporter ATP7A mediates resistance to cisplatin, carboplatin and oxaliplatin in ovarian cancer cells. Clin Cancer Res 10: 4661-4669, 2004.

5. Beretta GL, Gatti L, Tinelli S, et al: Cellular pharmacology of cisplatin in relation to the expression of human copper transporter CTR1 in different pairs of cisplatin-sensitive and -resistant cells. Biochem Pharmacol 68: 283-291, 2004.

6. Nakagawa M, Nomura Y, Kohno K, et al: Reduction of drug accumulation in cisplatin-resistant variants of human prostate cancer PC-3 cell line. J Urol 150: 1970-1973, 1993.

7. Katano K, Kondo A, Safaei R, et al: Acquisition of resistance to cisplatin is accompanied by changes in the cellular pharmacology of copper. Cancer Res 62: 6559-6565, 2002.

8. Safaei R and Howell SB: Copper transporters regulate the cellular pharmacology and sensitivity to Pt drugs. Crit Rev Oncol Hematol 53: 13-23, 2005.

9. Song IS, Savarj N, Siddik ZH, et al: Role of human copper transporter Ctrl in the transport of platinum-based antitumor agents in cisplatin-sensitive and cisplatin-resistant cells. Mol Cancer Ther 3: 1543-1549, 2004.

10. Holzer AK, Samimi G, Katano K, et al: The copper influx transporter human copper transport protein 1 regulates the uptake cisplatin in human ovarian carcinoma cells. Mol Pharmacol 66: 817-823, 2004.

11. Katano K, Safaei R, Samimi G, Holzer A, Rochdi M and Howell SB: The copper export pump ATP7B modulates the cellular pharmacology of carboplatin in ovarian carcinoma cells. Mol Pharmacol 64: 466-473, 2003.

12. Komatsu M, Sumizawa T, Mutoh M, et al: Copper-transporting P-type adenosine triphosphatase (ATP7B) is associated with cisplatin resistance. Cancer Res 60: 1312-1316, 2000.

13. Nakayama K, Miyazaki K, Kanzaki A, Fukumoto M and Takebayashi Y: Expression and cisplatin sensitivity of coppertransporting P-type adenosine triphosphatase (ATP7B) in human solid carcinoma cell lines. Oncol Rep 8: 1285-1287, 2001.

14. Kanzaki A, Toi M, Neamati N, et al: Copper-transporting P-type adenosine triphosphatase (ATP7B) is expressed in human breast carcinoma. Jpn J Cancer Res 93: 70-77, 2002.
15. Yamamoto E, Kohama G, Sunakawa H, Iwai M and Hiratsuka H: Mode of invasion, bleomycin sensitivity and clinical course in squamous cell carcinoma of the oral cavity. Cancer 51: 2175-2180, 1983.

16. Nozaki S, Endo Y, Nakahara H, et al: Inhibition of invasion and metastasis in oral cancer by targeting urokinase-type plasminogen activator receptor. Oral Oncol 41: 971-977, 2005.

17. Kohno K, Uchiumi T, Niina I, et al: Transcription factors and drug resistance. Eur J Cancer 41: 2577-2586, 2005.

18. Nakayama K, Kanzaki A, Ogawa K, Miyazaki K, Neamati N and Takebayashi Y: Copper-transporting P-type adenosine triphosphatase (ATP7B) as a cisplatin based chemoresistance marker in ovarian carcinoma: comparative analysis with expression of MDR1, MRP1, MRP2, LRP and BCRP. Int J Cancer 101: 488-495, 2002.

19. Borst P, Evers R, Kool M and Wijnholds J: The multidrug resistance protein family. Biochim Biophys Acta 1461: 347-357, 1999.

20. Hinoshita E, Uchiumi T, Taguchi K, et al: Increased expression of an ATP-binding cassette superfamily transporter, multidrug resistance protein 2 , in human colorectal carcinomas. Clin Cancer Res 6: 2401-2407, 2000.

21. Bentries-Alj M, Barbu V, Fillet M, et al: Inhibition of NF-кB transcription factor induces drug resistance through MDR 1 expression in cancer cells. Oncogene 22: 90-97, 2003.

22. Mabuchi S, Ohmichi M, Nishio Y, et al: Inhibition of NF-кB increases the efficacy of cisplatin in vitro and in vivo ovarian cancer models. J Biol Chem 279: 23477-23485, 2004.

23. Huber MA, Azoitei N, Baumann B, et al: NF- $\mathrm{kB}$ is essential for epithelial-mesenchymal transition and metastasis in a model of breast cancer progression. J Clin Invest 114: 569-581, 2004.

24. Huber MA, Beug $\mathrm{H}$ and Wirth T: Epithelial-mesenchymal transition: NF-kB takes center stage. Cell Cycle 3: 1477-1480, 2004.

25. Vincenti MP, Coon CL and Brinckerhoof CE: Nuclear factor $\kappa \mathrm{B} / \mathrm{p} 50$ activates an element in the distal matrix metalloproteinase 1 promptor in interleukin-1ß-stimulated synovial fibroblasts. Arthritis Rheum 41: 1987-1994, 1998.

26. Yang AD, Fan F, Camp ER, et al: Chronic oxaliplatin resistance induces epithelial-to-mesenchymal transition in colorectal cancer cell lines. Clin Cancer Res 12: 4147-4153, 2006.

27. Taki M, Kamata N, Yokoyama K, Fujimoto R, Tsutsumi S and Nagayama M: Down-regulation of Wnt-4 and up-regulation of Wnt-5a expression by epithelial-mesenchymal transition in human squamous carcinoma cells. Cancer Sci 94: 593-597, 2003. 